\title{
Validação brasileira do questionário de esquemas de Young: forma breve ${ }^{1}$
}

\author{
Brazilian validation of Young schema \\ questionnaire: short form
}

\author{
Milton José CAZASSA² \\ Margareth da Silva OLIVEIRA²
}

\begin{abstract}
Resumo
Esta pesquisa objetivou estudar as propriedades psicométricas da versão brasileira do questionário de esquemas de Young, forma reduzida, e mapear os esquemas cognitivos na amostra, buscando estabelecer correlações entre os níveis de ansiedade, depressão, desajustamento psicossocial e vulnerabilidade com os esquemas iniciais desadaptativos. A metodologia utilizada foi quantitativa, sendo a amostra da pesquisa constituída por 372 participantes. Os instrumentos utilizados foram um Questionário de Dados Sociodemográficos, o Questionário de Esquemas de Young - versão breve - e a Escala Fatorial de Ajustamento Emocional/Neuroticismo. Os resultados achados demonstraram a existência de validade na versão brasileira do Questionário de Esquemas de Young (forma breve) com relação à amostra estudada. Os resultados apontaram para o satisfatório grau de confiabilidade $(\alpha=0,955)$ e para a capacidade de discriminação do questionário, assim como para a validade concorrente com relação à escala fatorial de ajustamento emocional/neuroticismo.
\end{abstract}

Unitermos: Esquemas. Psicométria. Questionário de esquemas de Young. Terapia.

\begin{abstract}
The aim of this research was to study the psychometric properties of the Brazilian version of the Young Schema Questionnaire (short form) and map the cognitive schemas in the sample by establishing correlations between Early Maladaptive Schemas and variables such as anxiety levels, depression, lack of psychosocial adaptation and vulnerability. The methodology was quantitative. The sample comprised 372 subjects. A Demographic Questionnaire, Young Schema Questionnaire (short form) and Factorial Scale of Emotional Adjustment/Neuroticism were used as the instruments of assessment. The findings demonstrate the validity of the Brazilian version of the Young Schema Questionnaire (short form) for this group. The results showed a satisfactory degree of reliability $(\alpha=0.955)$ and the questionnaire's ability to make distinctions, as well as the convergent validity with regard to the Factorial Scale of Emotional Adjustment/Neuroticism.
\end{abstract}

Uniterms: Schemas. Psychometrics. Young schema questionnaire. Therapy.

$\boldsymbol{\nabla \nabla} \boldsymbol{\nabla} \boldsymbol{\nabla}$

1 Artigo elaborado a partir da dissertação de M.J. CAZASSA, intitulada "Mapeamento de esquemas cognitivos: validação da versão brasileira do Young Schema Questionnaire Short Form". Pontifícia Universidade Católica do Rio Grande do Sul, 2007. Apoio: Coordenação de Aperfeiçoamento de Pessoal de Nível Superior.

2 Pontifícia Universidade Católica do Rio Grande do Sul, Faculdade de Psicologia, Programa de Pós-Graduação em Psicologia. Av. Ipiranga 6681, Prédio 11, $9 \circ$ andar, 90619-900, Porto Alegre, RS, Brasil. Correspondência para/Correspondence to: M.J. CAZASSA. E-mail: <marga@pucrs.br>. 
A busca de possibilidades de tratamento capazes de contribuir efetivamente para a superação dos transtornos de personalidade e dos comportamentos dependentes é uma constante no processo evolutivo da humanidade. Conhecimentos provindos de diversas áreas têm oferecido estímulos para a construção de uma visão mais profunda e significativa do ser humano.

A terapia cognitiva para transtornos de personalidade - uma abordagem focada no esquema - caracteriza-se como um desses modelos clínicos e tem como precursores Jeffrey E. Young, Janet Klosko e Marjorie Weishaar. É considerada como um modelo integrativo de terapia que busca ampliar os referenciais para melhor atender à complexidade humana (Young, Klosko \& Weishaar, 2003).

Na visão de Young (2003), a terapia cognitiva tradicional (Beck, 1997; Beck, Rush, Shaw \& Emery, 1979) mostra-se limitada para o tratamento de pacientes com transtornos de personalidade. Young explica que esses pacientes não conseguem responder a algumas suposições fundamentais da terapia cognitiva de curto prazo, a saber: acesso, a partir de breve treinamento, a sentimentos, pensamentos e imagens; condição para identificar e delimitar problemas; motivação para aprender estratégias de autocontrole e realizar tarefas; engajamento em relação colaborativa com o psicoterapeuta, entre outros.

Assim, de acordo com Young (2003), a terapia focada em esquemas para o tratamento de transtornos da personalidade representa uma evolução do modelo cognitivo de Aaron Beck e tem como principal ênfase um nível mais aprofundado de cognição denominado de Esquema Inicial Desadaptativo (EID). Os EID são entendidos como estruturas estáveis e duradouras que se desenvolvem e se cristalizam precocemente na personalidade e/ou ao longo da vida do sujeito e que se encontram associados a diversas psicopatologias. Trata-se de padrões emocionais e cognitivos desadaptativos que tendem a se repetir no decorrer da vida, configurando processos de funcionamento da personalidade que mediam a interação do indivíduo com a realidade e que deverão ser mapeados e trabalhados a partir da relação estabelecida no processo psicoterapêutico (Cazassa \&Oliveira, 2008; Falcone \&Ventura, 2008; Falcone,

24 Gil \& Ferreira, 2007).
Os EID, em sua grande maioria, são causados pela vivência de experiências tóxicas que se repetem com alguma regularidade e que impossibilitam o preenchimento de necessidades emocionais essenciais do ser humano (vínculo seguro com outras pessoas, incluindo proteção, estabilidade e segurança; autonomia, competência e senso de identidade; liberdade para expressar necessidades e emoções; espontaneidade e diversão; e limites precisos e autocontrole). Apesar de nem todos os esquemas apresentarem traumas em sua origem, esses padrões de funcionamento são destrutivos e causadores de sofrimento, o que implica prejuízos na qualidade de vida das pessoas (Cazassa, 2007; Cazassa \& Oliveira, 2008; Irigaray \& Trentini, 2009; Kristensen, Schaefer \& Busnello, 2010; Schmidt, Joiner Jr., Young \& Telch, 1995; Young et al., 2003; Young, Klosko \& Weishaar, 2008).

Com o objetivo de mapear os EID, Young (2003) desenvolveu um instrumento chamado Young Schema Questionnaire (YSQ). O presente trabalho buscou verificar a aplicabilidade desses construtos na realidade brasileira, considerando a escassez de estudos empíricos no Brasil sobre o tema e a crescente utilização desse modelo nos outros países (Ball, Cobb-Richardson, Connolly, Bujosa \& O'Neall, 2005; Dutra, Callahan, Forman, Mendelsohn \& Herman, 2008; Harris \& Curtin, 2002; Pinto-Gouveia, Castilho, Galhardo \& Cunha, 2006; Rijkeboer, Bergh \& Bout, 2005; Riso et al., 2006; T. Saariaho, A. Saariaho, Karila \& Joukama, 2009; Soygüt, Karaosmanoğlu \& Çakir, 2009; Specht, Chapman \& Cellucci, 2009; Wright, Crawford \& Del Castillo 2009).

Assim sendo, o presente artigo apresenta um estudo empírico que utilizou o YSQ para verificação dos EID numa amostra brasileira. O instrumento foi aplicado juntamente com a escala fatorial de ajustamento emocional/neuroticismo (EFN) e um questionário de dados sociodemográficos. O objetivo principal foi realizar o estudo das propriedades psicométricas do instrumento, contemplando verificação da consistência interna da escala (alpha de Cronbach), validades convergentes e discriminantes. Os objetivos secundários estiveram ligados ao mapeamento dos esquemas cognitivos na amostra, buscando estabelecer correlações entre os níveis de ansiedade, depressão, desajustamento psicossocial e vulnerabilidade com os EID. 


\section{Método}

\section{Participantes}

A amostra da pesquisa foi constituída por 372 participantes da população em geral, na faixa etária entre 18 e 60 anos e que tinham estudado, no mínimo, até a $5^{2}$ série do ensino fundamental.

\section{Instrumentos}

Para a coleta de dados foram utilizados três instrumentos:

1) Questionário de dados sociodemográficos: construído com o intuito de mapear características sociodemográficas da amostra;

2) Young Schema Questionnaire (YSQ-S2): objetiva avaliar 15 EID considerados como centrais na cognição humana (Young, 2003). Formado por 75 afirmativas, tem uma escala tipo Likert de 1 a 6 para pontuação de acordo com a percepção do examinando. Os 15 esquemas encontram-se inseridos em cinco grandes domínios, a saber: desconexão/rejeição, autonomia/desempenho prejudicados, limites prejudicados, orientação para o outro e supervigilância/inibição.

O primeiro domínio, desconexão e rejeição, encontra-se ligado a sentimentos de frustração vivenciados pelas pessoas com relação às expectativas de segurança, estabilidade, carinho, empatia, aceitação e consideração. Os cinco esquemas vinculados a esse domínio são: privação emocional, abandono, desconfiança/abuso, isolamento social e defectividade/vergonha.

O segundo domínio, autonomia e desempenho prejudicados, avalia sentimentos de incapacidade experimentados pelos indivíduos no que tange à possibilidade de conquistar a autonomia necessária para sobreviver de forma independente e com bom desempenho (os esquemas são fracasso, dependência/incompetência, vulnerabilidade a dores e doenças, emaranhamento).

O terceiro, limites prejudicados, vincula-se à deficiência no estabelecimento de limites, seja pela ausência de responsabilidade com os demais e/ou pela dificuldade de concretizar objetivos. É caracterizado por prejuízos quanto a respeitar os direitos dos outros, a cooperar e a se comprometer com metas ou desafios. Os esquemas associados a esse domínio são os de merecimento e autocontrole/autodisciplina insuficientes.

O domínio seguinte, orientação para o outro, refere-se a um funcionamento que ocasiona um foco excessivo nos desejos e sentimentos dos outros, geralmente associado à constante busca de amor. Muitas vezes, a pessoa suplanta as próprias necessidades com o intuito de obter aprovação, podendo suprimir sua consciência, sentimentos e inclinações naturais. Os esquemas de subjugação e de autossacrifício compõem esse grupo.

O quinto e último domínio, supervigilância e inibição, está ligado ao bloqueio da felicidade, autoexpressão, relaxamento e relacionamentos íntimos, podendo gerar comprometimentos na própria saúde devido à ênfase excessiva na supressão dos sentimentos, dos impulsos e das escolhas pessoais espontâneas. As regras e expectativas rígidas internalizadas sobre desempenho e comportamento ético geralmente integram esse padrão de funcionamento. Inibição emocional e padrões inflexíveis são os dois esquemas que integram esse contexto.

A tradução do material para a língua portuguesa foi realizada por Maria Adriana Veríssimo Veronese para a versão brasileira do livro de Young (2003). Apesar de, atualmente, não existir tradução, para o português, da forma breve do questionário de esquemas, verificou-se que essa forma é composta por questões idênticas às utilizadas na forma longa.

A observância da equivalência total entre as versões do instrumento (forma longa e forma breve), visitadas em sua língua materna, oportunizaram o aproveitamento da tradução existente no Brasil (Young, 2003). Assim, o instrumento utilizado seguiu, também, as mesmas instruções existentes na forma longa.

Além disso, foi proposta uma adaptação da escala de avaliação do questionário após submetimento à apreciação de dois juízes e aplicação em grupo-piloto composto por cinco psicólogos, alunos de pós-graduação, e um professor doutor do curso de psicologia. Os juízes foram profissionais especialistas no âmbito da validação de instrumentos psicológicos, todos professores de pós-graduação, não tendo existido qualquer contato entre eles. 
Com relação ao grupo-piloto, os participantes foram convidados a tecer críticas e comentários acerca das instruções de aplicação e sobre os 75 itens. As críticas deram origem a uma alteração nas instruções com o intuito de facilitação da leitura e de melhoramento da compreensão. Os 75 itens não sofreram alteração.

3) Escala fatorial de ajustamento emocional/ neuroticismo: Trata-se de um instrumento que avalia uma dimensão da personalidade humana denominada fator $\mathrm{N}$, também chamado de neuroticismo. Essa medida refere-se ao nível de ajustamento e estabilidade emocional do indivíduo, os quais se encontram associados a desconfortos psicológicos e aos estilos cognitivos e comportamentais decorrentes de um padrão de funcionamento. O questionário é composto por 82 itens divididos em 4 escalas: vulnerabilidade, desajustamento psicossocial, ansiedade e depressão.

\section{Procedimentos}

Este projeto foi aprovado pela comissão científica da Faculdade de Psicologia e pelo comitê de ética em pesquisa da PUCRS (CEP 07/03471).

A abordagem dos participantes seguiu os critérios da amostra por conveniência e ocorreu a partir do contato direto realizado pela equipe de pesquisadores, que foi treinada para conduzir rapport padronizado junto aos participantes. As aplicações ocorreram de forma individual e coletiva.

O início do preenchimento dos questionários era precedido pela leitura e assinatura do termo de consentimento, o qual continha as informações sobre a pesquisa e oferecia os contatos dos pesquisadores e do comitê de ética da PUCRS.

Os dados foram processados no programa estatístico 12.0 Program Statistical Package for the Social Sciences (SPSS). Foram realizadas análises descritivas e de distribuição de frequências para demonstração do perfil dos participantes da pesquisa. Recorreu-se ao teste do Qui-quadrado para verificação da homogeneidade da amostra quanto às variáveis sexo, escolaridade, estado civil e renda. A variável numérica'idade' e os 75 itens do YSQ foram submetidos ao teste de normalidade Kolmogorov-Smirnov, tendo em vista o tamanho amos-

26 tral (>30 participantes).
As análises de fidedignidade, com o intuito de avaliar a consistência interna da escala, foram encaminhadas por meio da análise de confiabilidade e da verificação do coeficiente Alpha de Cronbach. Os estudos contemplaram a escala global, ou seja, todos os itens incluídos, tanto para o YSQ quanto para a EFN.

Os resultados vinculados à homogeneidade e normalidade indicaram a necessidade de utilização de estatísticas não paramétricas, tendo sido empregada a técnica da correlação de Spearman para verificação de existência de correlação entre os instrumentos utilizados no estudo (validade concorrente).

O teste de Kruskal-Wallis foi empregado para verificar as diferenças entre os grupos abaixo da média, na média e acima da média, estratificados a partir dos escores da "vulnerabilidade", "desajustamento psicossocial", "ansiedade" e "depressão" da EFN com relação aos 15 EID, extraídos a partir do YSQ.

\section{Resultados}

A amostra foi constituída por 372 participantes - $30,9 \%$ do sexo masculino e $69,1 \%$ do sexo feminino -, residentes na Grande Porto Alegre (RS). A idade média foi de 28,5 anos ( $D P=10,14)$, sendo a idade mínima 18 e a máxima 60 anos. No que tange à variável escolaridade, os percentuais evidenciaram 6,0\% dos participantes com Ensino Fundamental, 48,1\% com Ensino Médio Completo, 34,4\% com Ensino Superior, 9,2\% com Pós-Graduação. Como omissão dessa informação, tivemos o percentual de $2,4 \%$.

\section{Consistência interna do YSQ-S2 e da EFN}

O coeficiente Alpha de Cronbach foi utilizado para verificação da consistência interna do questionário de esquemas de Young - forma breve (YSQ-S2), considerando-se os 15 esquemas e a escala global. Para os 75 itens, o alpha foi 0,955, o que sugere excelente grau de consistência interna.

No que tange aos 15 EID, com exceção do esquema de dependência/incompetência $(0,566)$, todos os demais apresentaram satisfatórios níveis de consistência interna, variando de 0,719 a 0,905, a saber:" privação emocional" (0,856), "abandono" (0,855), "desconfiança/ 
abuso" (0,779), "isolamento social" (0,719), "defectividade/vergonha" $(0,796)$, "fracasso" $(0,905)$, "vulnerabilidade a dores e doenças" (0,802), "emaranhamento" (0,815), "subjugação" (0,779), "autossacrifício" $(0,851)$, "inibição emocional" (0,875), "padrões inflexíveis" $(0,742)$, "merecimento" $(0,753)$ e "autocontrole e autodisciplina insuficientes" $(0,808)$.

Quanto à EFN, verificou-se que o alpha de cronbach foi de 0,953 para a escala global (82 itens). Nas medidas das subescalas, tivemos os seguintes coeficientes de alpha: "vulnerabilidade", 0,920, "desajustamento psicossocial", 0,796, "ansiedade", 0,898 e "depressão", 0,836, o que evidencia que esse instrumento apresenta, também, satisfatório grau de confiabilidade na amostra, o que confirma achados de Hutz e Nunes (2001).

\section{Validade concorrente}

O estudo de correlação envolveu os 15 EID e a pontuação geral da EFN.

Os 15 EID apresentaram níveis de correlação positiva altamente significativos em relação à pontuação geral da EFN. Tais resultados oferecem evidências para o atendimento de um critério importante para a validade concorrente do YSQ-S2. Assim, quanto mais disfuncionais apresentam-se os esquemas, maior tende a ser a pontuação nas dimensões da "vulnerabilidade", "desajustamento psicossocial","ansiedade"e "depressão" da EFN.

Em contrapartida, quanto mais reduzida a pontuação no YSQ-S2, menor propende a ser a pontuação na EFN (Tabela 1).

\section{Propriedades discriminantes do YSQ-S2}

Para o estudo das propriedades discriminantes do YSQ-S2, realizou-se uma estratificação das medidas da EFN. As escalas de "vulnerabilidade","desajustamento psicossocial", "ansiedade" e "depressão" foram categorizadas em abaixo da média, na média e acima da média. Quanto maior a pontuação do participante em cada uma das escalas da EFN, maior o grau de neuroticismo (fator N) e de instabilidade emocional do indivíduo (Hutz \&Nunes, 2001).
Tabela 1. Coeficientes de correlação entre os esquemas iniciais desadaptativos e a pontuação geral da escala fatorial de ajustamento emocional/neuroticismo.

\begin{tabular}{lc}
\hline N=343 & $\begin{array}{c}\text { Coeficiente } \\
\text { Correlação }\end{array}$ \\
\hline Privação emocional & $p$ \\
Abandono & 0,501 \\
Desconfiança/abuso & 0,569 \\
Isolamento social & 0,532 \\
Defectividade/vergonha & 0,556 \\
Fracasso & 0,539 \\
Dependência/incompetência & 0,507 \\
Vulnerabilidade a dores e doenças & 0,463 \\
Emaranhamento & 0,548 \\
Subjugação & 0,000 \\
Autossacrifício & 0,472 \\
Inibição emocional & 0,527 \\
Padrões inflexíveis & 0,354 \\
Merecimento & 0,464 \\
Autocontrole e autodisciplina insuficientes & 0,381 \\
\hline
\end{tabular}

Assim, a suposição é a de que as pessoas que apresentam índices acima da média na EFN devam apresentar, também, pontuações mais elevadas no YSQ-S2, o que significaria uma maior disfuncionalidade nos esquemas cognitivos. Caso essa hipótese fique evidenciada, torna-se possível afirmar que o instrumento desenvolvido por Jeffrey E. Young tem adequação quanto ao grau de senbilidade para a amostra estudada.

São apresentadas tabelas que demonstram como as médias de cada esquema ficaram distribuídas, considerando-se o critério de estratificação abaixo da média, na média e acima da média para os fatores"vulnerabilidade", "desajustamento psicossocial", "ansiedade"e "depressão", da EFN.

Constataram-se diferenças significativas ao nível de $p<0,001$. Esses achados significam que as pessoas com pontuações acima da média no quesito "vulnerabilidade" da EFN apresentaram também pontuações mais elevadas no YSQ-S2, com diferenças estatísticas significativas entre os grupos acima da média, na média e abaixo da média, assim estratificados pela pontuação da EFN (Tabela 2).

Observa-se a existência de diferenças significativas para todos os esquemas, considerando-se a dimensão do "desajustamento psicossocial" da EFN. Assim, os participantes alocados abaixo da média, na 
média e acima da média apresentaram médias significativamente distintas, sempre num sentido ascendente, isto é, quanto maior a pontuação na EFN também mais elevada a pontuação no YSQ (Tabela 3).

Nessa perspectiva, apesar de as quatro escalas "vulnerabilidade", "desajustamento psicossocial", "ansiedade"e "depressão" apresentarem elevada correlação positiva com os EID, a escala de desajustamento psicossocial é a que evidenciou menores, porém significativos, graus de correlação positiva (Tabelas 4 e 5).

\section{Discussão}

Os achados revelaram satisfatórios graus de consistência interna da escala, confirmando resultados apresentados em outros países (Baranoff, Oei, Cho \& Kwon 2006; Hoffart et al., 2005; Lee Taylor \& Dunn, 1999; Schmidt et al., 1995; Waller Meyer \& Ohanian, 2001; Welburn, Coristine, Dagg, Pontefract \& Jordan, 2002). No presente estudo, excetuando-se o esquema de dependência/incompetência $(0,566)$, todos os demais níveis de consistência interna variaram de 0,719 a 0,905.

Tabela 2. Comparação entre as médias dos diferentes graus de "vulnerabilidade" medidos a partir da escala fatorial de ajustamento emocional/ neuroticismo com relação aos esquemas iniciais desadaptativos.

\begin{tabular}{|c|c|c|c|c|c|}
\hline $\mathrm{N}=344$ & <Média (n=190) & Média $(n=70)$ & >Média $(\mathrm{n}=84)$ & Kruskal-Wallis $\left(\chi^{2}\right)$ & $p$ \\
\hline Privação emocional & 141,20 & 192,84 & 226,35 & 47,81 & \\
\hline Abandono & 131,78 & 200,60 & 241,18 & 78,03 & \\
\hline Desconfiança/abuso & 137,08 & 195,36 & 233,58 & 60,02 & \\
\hline Isolamento social & 134,91 & 184,57 & 247,46 & 77,35 & \\
\hline Defectividade/vergonha & 140,13 & 189,03 & 231,94 & 70,04 & \\
\hline Fracasso & 131,83 & 190,69 & 249,33 & 88,74 & \\
\hline Dependência/incompetência & 136,27 & 197,69 & 233,45 & 63,90 & \\
\hline Vulnerabilidade a dores e doenças & 134,00 & 205,01 & 232,49 & 67,62 & 0,000 \\
\hline Emaranhamento & 134,13 & 205,80 & 231,54 & 67,99 & \\
\hline Subjugação & 124,03 & 202,36 & 257,24 & 114,60 & \\
\hline Autossacrifício & 139,31 & 205,68 & 219,92 & 48,20 & \\
\hline Inibição emocional & 137,23 & 185,54 & 241,42 & 67,07 & \\
\hline Padrões inflexíveis & 142,91 & 187,46 & 226,96 & 43,74 & \\
\hline Merecimento & 134,23 & 201,59 & 234,82 & 67,43 & \\
\hline Autocontrole e autodisciplina insuficientes & 124,38 & 209,31 & 250,67 & 106,71 & \\
\hline
\end{tabular}

Tabela 3. Comparação entre as médias dos diferentes graus de "desajustamento psicossocial" medidos a partir da escala fatorial de ajustamento emocional/neuroticismo com relação aos esquemas iniciais desadaptativos.

\begin{tabular}{|c|c|c|c|c|c|c|}
\hline & $\mathrm{N}=344$ & <Média $(\mathrm{n}=179)$ & Média (n=87) & $>$ Média $(\mathrm{n}=78)$ & Kruskal-Wallis $\left(\chi^{2}\right)$ & $p$ \\
\hline \multirow{7}{*}{ 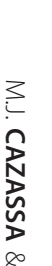 } & Privação mocional & 146,54 & 200,07 & 201,32 & 26,23 & \multirow{10}{*}{0,000} \\
\hline & Abandono & 147,51 & 183,06 & 218,07 & 28,86 & \\
\hline & Desconfiança/abuso & 135,73 & 210,78 & 214,20 & 51,53 & \\
\hline & Isolamento social & 144,92 & 197,26 & 208,16 & 29,75 & \\
\hline & Defectividade/vergonha & 148,71 & 193,18 & 204,02 & 29,37 & \\
\hline & Fracasso & 151,94 & 188,33 & 202,02 & 17,61 & \\
\hline & Dependência/incompetência & 145,32 & 197,03 & 207,52 & 29,56 & \\
\hline 3 & Vulnerabilidade a dores e doenças & 141,87 & 194,41 & 218,36 & 38,41 & \\
\hline \multirow{4}{*}{ 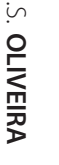 } & Emaranhamento & 150,59 & 192,11 & 200,91 & 19,07 & \\
\hline & Subjugação & 148,32 & 194,20 & 203,79 & 22,88 & \\
\hline & Autossacrifício & 159,82 & 184,57 & 188,13 & 6,13 & 0,046 \\
\hline & Inibição emocional & 149,71 & 194,23 & 200,56 & 20,24 & 0,000 \\
\hline 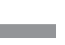 & Padrões inflexíveis & 153,89 & 187,51 & 198,47 & 13,61 & 0,001 \\
\hline & Merecimento & 137,70 & 195,18 & 227,06 & 50,17 & \multirow{2}{*}{0,000} \\
\hline 28 & Autocontrole e autodisciplina insuficientes & 135,07 & 198,57 & 229,31 & 57,18 & \\
\hline
\end{tabular}


Tabela 4. Comparação entre as médias dos diferentes graus de "ansiedade" medidos a partir da escala fatorial de ajustamento emocional/ neuroticismo com relação aos esquemas iniciais desadaptativos.

\begin{tabular}{|c|c|c|c|c|c|}
\hline$N=344$ & <Média $(n=191)$ & Média $(n=66)$ & $>$ Média $(n=87)$ & Kruskal-Wallis $\left(\chi^{2}\right)$ & $p$ \\
\hline Privação emocional & 138,99 & 183,80 & 237,48 & 61,53 & \\
\hline Abandono & 131,91 & 188,11 & 249,77 & 86,55 & \\
\hline Desconfiança/abuso & 135,09 & 188,07 & 242,81 & 72,77 & \\
\hline Isolamento social & 140,68 & 172,13 & 242,64 & 64,04 & \\
\hline Defectividade/vergonha & 144,33 & 181,61 & 227,44 & 57,07 & \\
\hline Fracasso & 144,24 & 186,27 & 224,10 & 42,25 & \\
\hline Dependência/incompetência & 140,21 & 198,13 & 223,94 & 49,85 & \\
\hline Vulnerabilidade a dores e doenças & 139,20 & 177,93 & 241,49 & 64,53 & 0,000 \\
\hline Emaranhamento & 140,21 & 192,78 & 227,99 & 51,68 & \\
\hline Subjugação & 143,47 & 182,10 & 228,94 & 45,78 & \\
\hline Autossacrifício & 147,32 & 185,23 & 218,11 & 31,71 & \\
\hline Inibição emocional & 139,21 & 205,36 & 220,65 & 50,21 & \\
\hline Padrões inflexíveis & 139,77 & 192,04 & 229,53 & 52,02 & \\
\hline Merecimento & 133,33 & 194,65 & 241,69 & 75,39 & \\
\hline Autocontrole e autodisciplina insuficientes & 131,95 & 197,56 & 242,52 & 79,62 & \\
\hline
\end{tabular}

Tabela 5. Comparação entre as médias dos diferentes graus de "depressão" medidos a partir da escala fatorial de ajustamento emocional/ neuroticismo com relação aos esquemas iniciais desadaptativos.

\begin{tabular}{|c|c|c|c|c|c|}
\hline $\mathrm{N}=344$ & $<$ Média $(\mathrm{n}=118)$ & Média $(n=106)$ & >Média $(n=120)$ & Kruskal-Wallis $\left(\chi^{2}\right)$ & $p$ \\
\hline Privação emocional & 128,25 & 159,24 & 227,73 & 64,18 & \multirow{10}{*}{0,000} \\
\hline Abandono & 131,75 & 168,25 & 216,33 & 43,61 & \\
\hline Desconfiança/abuso & 143,19 & 159,63 & 212,69 & 31,91 & \\
\hline Isolamento social & 126,32 & 153,01 & 235,12 & 78,57 & \\
\hline Defectividade/vergonha & 135,39 & 149,74 & 229,10 & 81,87 & \\
\hline Fracasso & 129,77 & 157,65 & 227,63 & 64,29 & \\
\hline Dependência/incompetência & 136,39 & 159,12 & 219,83 & 46,59 & \\
\hline Vulnerabilidade a dores e doenças & 134,09 & 166,97 & 215,15 & 40,65 & \\
\hline Emaranhamento & 141,04 & 164,31 & 210,67 & 31,24 & \\
\hline Subjugação & 144,45 & 148,04 & 221,69 & 46,03 & \\
\hline Autossacrifício & 154,27 & 161,12 & 200,48 & 14,89 & 0,001 \\
\hline Inibição emocional & 139,49 & 155,81 & 219,70 & 44,07 & 0,000 \\
\hline Padrões inflexíveis & 155,49 & 166,84 & 194,23 & 9,55 & 0,008 \\
\hline Merecimento & 151,07 & 156,28 & 207,90 & 23,62 & \multirow{2}{*}{0,000} \\
\hline Autocontrole e autodisciplina insuficientes & 127,59 & 157,41 & 229,99 & 67,07 & \\
\hline
\end{tabular}

No que tange às análises das propriedades discriminantes do instrumento, muito embora o estudo não tenha trabalhado diretamente com grupos clínicos e não clínicos, buscou-se, a partir de uma estratificação da EFN, a conformação de grupos distintos. As divisões realizadas em abaixo da média, na média e acima da média nas 4 escalas da EFN ("vulnerabilidade", "desajustamento psicossocial", "ansiedade"e "depressão") puderam demonstrar que o grupo que pontuou acima da média apresentou, também, medidas significativamente mais elevadas em todos os EID quando comparado aos participantes que tiveram escores abaixo da média ou na média. Isso significa que os participantes da pesquisa que apresentaram o fator $\mathrm{N}$ (neuroticismo) pela EFN (grupo acima da média) demonstraram, também, maior disfuncionalidade nos EID.

Da mesma forma, o estudo de correlação demonstrou índices significativos de correlação positiva entre os dois instrumentos utilizados na pesquisa, ou seja, quanto maior a pontuação no YSQ, mais elevada a pontuação na EFN.

Os resultados obtidos na amostra encontram consonância com estudos internacionais, os quais de- 
monstraram significativos graus de correlação positiva do YSQ com outros instrumentos de medida utilizados como padrão-ouro, bem como apontaram para diferenças significativas entre as medidas obtidas para os grupos clínicose não clínicos (Anderson, Rieger \& Caterson, 2006; Calvete, Estévez, Arroyabe \& Ruiz, 2005; Rijkeboer, Bergh \& Bout, 2005; Waller et al., 2001; Welburn et al., 2002).

Tais achados ofereceram indicativos para a validade concorrente do instrumento, tendo em vista que maiores níveis de neuroticismo estão associados a pessoas mais propensas à vivência de sofrimento emocional (Hutz \& Nunes, 2001) ou, em outras palavras, com maior propensão à disfuncionalidade dos esquemas. Dessa forma, os resultados apontaram para o fato de que o YSQ-S2 tem satisfatórias propriedades discriminativas na amostra estudada.

\section{Considerações Finais}

Os objetivos desta pesquisa foram estudar as propriedades psicométricas da versão brasileira do YSQ-S2, e mapear esquemas cognitivos na amostra, buscando estabelecer correlações entre os níveis de ansiedade, depressão, desajustamento psicossocial e vulnerabilidade com os EID.

De modo geral, os achados da presente pesquisa ofereceram subsídios para a avaliação de quesitos que demonstraram a existência de validade na versão brasileira do YSQ-S2 para a amostra estudada. Os resultados apontaram para o satisfatório grau de fidedignidade e para a capacidade de discriminação do questionário, assim como para a validade concorrente com relação à EFN.

Considera-se fundamental salientar a importância da realização de novos estudos que visem a aprofundar a verificação da aplicabilidade do YSQ-S2 (forma breve) na realidade brasileira, principalmente no que tange à discriminação entre populações clínicas e não clínicas, bem como na aplicação de teste e reteste para verificação da estabilidade temporal do instrumento.

\section{Referências}

Anderson, K., Rieger, E., \& Caterson, I. (2006). A comparison 30 of maladaptive schemata in treatment-seeking obese adults and normal-weight control subjects. Journal of Psychosomatic Research, 60 (3), 245-252.

Ball, S. A., Cobb-Richardson, P., Connolly, A. J., Bujosa, C. T., \& O'Neall, T. W. (2005). Substance abuse and personality disorders in homeless drop-in center clients: symptom severity and psychotherapy retention in a randomized clinical trial. Comprehensive Psychiatry, 46 (5), 371-379.

Baranoff, J., Oei, T. P. S., Cho, S. H., \& Kwon, S. M. (2006). Factor structure and internal consistency of the Young Schema Questionnaire (Short Form) in Korean and Australian samples. Journal of Affective Disorders, 93 (1-3), 133-140.

Beck, A. T., Rush, A. J., Shaw, B. Y., \& Emery, G. (1979). Cognitive therapy of depression. New York: Guilford.

Beck, J. (1997). Terapia cognitiva: teoria e prática. Porto Alegre: Artes Médicas.

Calvete, E., Estévez, A., Arroyabe, E. L., \& Ruiz, P. (2005). The Schema Questionnaire - Short Form: structure and relationship with automatic thoughts and symptoms of affective disorders. European Journal of Psychological Assessment, 21 (2), 90-99.

Cazassa, M. J. (2007). Mapeamento de esquemas cognitivos: validação da versão brasileira do Young Schema Questionnaire - Short Form. Dissertação de mestrado não-publicada, Pontifícia Universidade Católica do Rio Grande do Sul, Porto Alegre.

Cazassa, M. J., \& Oliveira, M. S. (2008). Terapia focada em esquemas: conceituação e pesquisas. Revista de Psiquiatria Clínica, 35 (5), 187-195.

Dutra, L., Callahan, K., Forman, E., Mendelsohn, M., \& Herman, J. (2008). Core schemas and suicidality in a chronically traumatized population. The Journal of Nervous and Mental Disease, 196 (1), 71-74.

Falcone, E. M. O., Gil, D. B., \& Ferreira, M. C. (2007). Um estudo comparativo da frequência de verbalização empática entre psicoterapeutas de diferentes abordagens teóricas. Estudos de Psicologia (Campinas), 24 (4), 451-461. doi: 10.1590/S0103-166X2007000400005.

Falcone, E. M. O., \& Ventura, P. R. (2008). Entrevista com Dr. Jeffrey Young. Revista Brasileira de Terapias Cognitivas, 4 (1), $1-7$.

Harris, A. E., \& Curtin, L. (2002). Parental perceptions, early maladaptive schemas, and depressive symptoms in young adults. Cognitive Therapy and Research, 26 (3), 405-416.

Hoffart, A., Sexton, H., Hedley, L. M., Wang, C. E., Holthe, H., Haugum, J. A., et al. (2006). The structure of maladaptive schemas: a confirmatory factor analysis and a psychometric evaluation of factor-derived scales. Cognitive Therapy and Research, 29 (6), 627-644.

Hutz, C., \& Nunes, C. H. S. S. (2001). Escala fatorial de ajustamento emocional/neuroticismo: EFN. São Paulo: Casa do Psicólogo.

Irigaray, T. Q., \& Trentini, C. M. (2009). Qualidade de vida em idosas: a importância da dimensão subjetiva. Estudos de Psicologia (Campinas), 26 (3), 297-304. doi: 10.1590/S0 103-166X2009000300003. 
Kristensen, C. H., Schaefer, L. S., \& Busnello, F. B. (2010). Estratégias de coping e sintomas de stress na adolescência. Estudos de Psicologia (Campinas), 27 (1), 21-30. doi: 10.15 90/S0103-166X2010000100003.

Lee, C. W., Taylor, G. \& Dunn, J. (1999). Factor structure of the schema questionnaire in a large clinical sample. Cognitive Therapy and Research, 23 (4), 441-451.

Pinto-Gouveia, J., Castilho, P., Galhardo, A., \& Cunha, M. (2006). Early maladaptive schemas and social phobia. Cognitive Therapy and Research, 30 (5), 571-584.

Rijkeboer, M. M., Bergh, H., \& Bout, J. (2005). Stability and discriminative power of the Young schema-questionnaire in a Dutch clinical versus non-clinical population. Journal of Behavior Therapy and Experimental Psychiatry, 36 (2), 129-144.

Riso, L. P., Froman, S. E., Raouf, M., Gable, P., Maddux, R. E., Turini-Santorelli, N., et al. (2006). The long-term stability of early maladaptive schemas. Cognitive Therapy and Research, 30 (4), 515-529.

Saariaho, T., Saariaho, A., Karila, I., \& Joukama, M. (2009). The psychometric properties of the finnish Young schema questionnaire in chronic pain patients and a non-clinical sample. Behavior Therapy and Experimental Psychiatry, 40 (1), 158-68.

Schmidt, N. B., Joiner Jr., T. E., Young, J. E., \& Telch, M. J. (1995). The schema questionnaire: investigation of psychometric properties and the hierarchical structure of a measure of maladaptive schemas. Cognitive Therapy and Research, 19 (3), 295-321.

Soygüt, G., Karaosmano ğlu, A., \& Çakir, Z. (2009). Assessment of early maladaptive schemas: a psychometric study of the Turkish Young Schema questionnaire-short form-3. Turkish Journal of Psychiatry, 20 (1), 75-84.
Specht, M. W., Chapman., \& Cellucci, T. (2009). Schemas and borderline personality disorder symptoms in incarcerated women. Journal of Behavior Therapy and Experimental Psychiatry, 40 (2), 256-264.

Waller, G., Meyer, C., \& Ohanian, V. (2001). Psychometric properties of the long and short versions of the Young Schema Questionnaire: core beliefs among bulimic and comparison women. Cognitive Therapy and Research, 25 (2), 137-147.

Welburn, K., Coristine, M., Dagg, P., Pontefract, A., \& Jordan S. (2002). The Schema questionnaire - short form: factor analysis and relationship between schemas and symptoms. Cognitive Therapy and Research, 26 (4), 519-530.

Wright, M.O., Crawford, E., \& Del Castillo, D. (2009). Childhood emotional maltreatment and later psychological distress among college students: the mediating role of maladaptive schemas. Child Abuse \& Neglect, 33 (1), 59-68

Young. J. E. (2003). Terapia cognitiva para transtornos da personalidade: uma abordagem focada em esquemas ( $3^{a}$ ed.). Porto Alegre: ArtMed.

Young, J. E., Klosko, J. S., \& Weishaar, M. E. (2003). Schema therapy: a practitioner's guide. New York: The Guilford Press.

Young, J. E., Klosko, J. S., \& Weishaar, M. E. (2008). Terapia do esquema: guia de técnicas cognitivo-comportamentais inovadoras. New York: The Guilford Press.

Recebido em: 20/7/2009

Versão final reapresentada em: 10/2/2011

Aprovado em: 4/11/2011 
